\title{
Histogram Model for 3D Textures
}

\author{
Kristin J. Dana and Shree K. Nayar \\ Department of Computer Science \\ Columbia University \\ New York, NY 10027
}

\begin{abstract}
Image texture can arise not only from surface albedo variations ( $2 D$ texture) but also from surface height variations ( $3 D$ texture). Since the appearance of $3 D$ texture depends on the illumination and viewing direction in a complicated manner. such image texture can be called a bidirectional texture function. A fundamental representation of image texture is the histogram of pixel intensities. Since the histogram of $3 D$ texture also depends on the illumination and viewing directions in a complex fashion, we refer to it as a bidirectional histogram. In this work. we present a concise analytical model for the bidirectional histogram of Lambertian, isotropic, randomly rough surfaces! which are common in real-world scenes. We demonstrate the accuracy of the histogram model by fitting to several samples from the ColumbiaUtrecht texture database. The parameters obtained from the model fits are roughness measures which can be used in texture recognition schemes. In addition. the model has potential application in estimating illumination direction in scenes where surfaces of known tilt and roughness are visible. We demonstrate the usefulness of our model by employing it in a novel $3 D$ texture synthesis procedure.
\end{abstract}

\section{Introduction}

The term image texture. or simply texture, usually refers to the digital image of a textured surface. In order to understand image texture, the nature of the surface texture must be specified. Image texture can arise not only from surface albedo variations (2D texture) but also from surface height variations (3D texture). The distinction between $3 \mathrm{D}$ texture and $2 \mathrm{D}$ texture is explored in recent work $[3],[8],[4],[7],[5]$. While there is a large body of work dealing with algorithms for the analysis and synthesis of $2 \mathrm{D}$ texture, comparable work for $3 \mathrm{D}$ texture is quite sparse. Since the appearance of 3D texture depends on the illumination and viewing direction in a complicated manner, it is useful to refer to image texture as a bidirectional texture function.

Modeling and synthesizing this bidirectional texture is key to achieving robust texture recognition and segmentation as well as photorealistic texture rendering. A fundamental representation of texture is the histogram of pixel intensities. For 3D texture, just as image texture is bidirectional, the histogram is a bidirectional histogram. Changes in the histogram of $3 D$ texture with illumination and viewing directions are indicative of the surface structure. The work of [8] also addresses histograms of 3D texture by investigating the physical mechanisms underlying bidirectional histograms from a large variety of surfaces and by using statistical simulations to generate histograms of gaussian rough surfaces.

In this work we develop an analytical model of the bidirectional histogram of image texture. For arbitrary surfaces, developing such a model is extremely difficult. So, for tractability, we assume the imaged surface has an isotropic random-slope profile and constant-albedo Lambertian reflectance. This model proves to be a good approximation for a variety of natural and man-made surfaces found in ordinary scenes. Our model is based on a geometric/ photometric analysis of the interaction of light with the surface. We show the accuracy of the model by fitting to the histograms of real $3 D$ textures from the Columbia-Utrecht texture database [3].

The model can be used in applications for both computer vision and computer graphics. The parameters obtained from the model fits are roughness measures which can be used in texture recognition schemes. In addition, the model has potential application in'estimating illumination direction in scenes where surfaces of known tilt and roughness are visible. We demonstrate the usefulness of our model by employing it in a novel 3D texture synthesis procedure called texture-morphing. We show that results obtained using texture-morphing are superior to those obtained with standard techniques of texture-mapping.

\section{Histogram Model}

There are two main tasks involved with the development of the histogram model. The first task is the conversion of the surface slope probability density function (pdf) to the image intensity pdf. This conversion requires a careful analysis of surface masking, shadowing and shading that results in an expression for the image intensity pdf in integral form. This integral is rather complicated and does not lend itself to an analytical solution. The second task of the model development is a suitable approximation of the integral that leads to a concise parametric histogram representation.

\subsection{Surface Normal PDF}

The surface is assumed to be isotropic with a gaussian distributed surface slope. Let $\mathrm{G}$ denote the global normal of the surface. The local surface normal is a random vector denoted by $\mathrm{N}$ with polar angle $\theta$ and azimuthal 
angle $\phi$ relative to $G$. The surface is such that $N$ takes values in the unit hemisphere where $\theta \in[0, \pi / 2]$ and $\phi \in[0,2 \pi]$. Let the vector $\gamma=\left(\gamma_{x}, \gamma_{y}\right)$ be the random surface slope with pdf given by

$$
p_{\gamma}\left(\gamma_{x}, \gamma_{y}\right)=\frac{1}{2 \pi \sigma^{2}} \exp \left(-\left(\gamma_{x}^{2}+\gamma_{y}^{2}\right) /\left(2 \sigma^{2}\right)\right)
$$

where the constant $\sigma$ is the standard deviation (surface roughness), and $\gamma_{x}, \gamma_{y}$ are the slopes in the $x, y$ directions of the cartesian coordinate frame whose $z$ axis is aligned with $G$. The probability of a particular surface slope $\left(\gamma_{x}, \gamma_{y}\right)$ with a corresponding surface normal $N$ is given by $p_{\gamma}\left(\gamma_{x}, \gamma_{y}\right) d \gamma_{x} d \gamma_{y}$, or, equivalently, by $p_{N}(\theta, \phi) \sin \theta d \theta d \phi$, where $p_{N}(\theta, \phi)$ is the pdf of the random surface normal $N$. The pdf $p_{N}(\theta, \phi)$ can be derived from $p_{\gamma}\left(\gamma_{x}, \gamma_{y}\right)$ using the Jacobian. Since $\gamma_{x}=\tan \theta \cos \phi$, and $\gamma_{y}=\tan \theta \sin \phi$, the determinant of the Jacobian is

$$
\left|\begin{array}{ll}
\frac{\partial \gamma_{x}}{\partial \theta} & \frac{\partial \gamma_{x}}{\partial \phi} \\
\frac{\partial \gamma_{y}}{\partial \theta} & \frac{\partial \gamma_{y}}{\partial \phi}
\end{array}\right|=\left(1+\tan ^{2} \theta\right) \tan \theta
$$

Therefore,

$$
p_{N}(\theta . \phi)=\frac{1}{2 \pi \sigma^{2}} \frac{\exp \left(-\tan ^{2} \theta /\left(2 \sigma^{2}\right)\right)}{\cos ^{3} \theta} .
$$

\subsection{Intensity PDF}

Now consider the image intensity represented by the random variable $I$, taking values in $[0,1]$. Assume that the albedo $\rho$ is unity; the generalization to arbitrary albedo is addressed in a later section. We assume orthographic projection and an imaging system where each pixel images a small planar surface patch. Consider a particular intensity $I_{o}$ from a surface illuminated by a distant source from the direction $S$. Under the Lambertian reflectance assumption, surface points with normals in the set $C_{o}=\left\{n: n \cdot S=I_{o}\right\}$ give rise to pixels of intensity $I_{o}$. The set $C_{o}$ corresponds to a cone oriented toward $S$, as illustrated in Figure 1. The probability of the occurrence of a surface normal which gives rise to intensity $I_{o}$ is the integral of the surface normal pdf, given by Equation 3, over this cone of surface normals $C_{o}$. Since the final goal is an expression for the histogram, probabilities must be considered in the image domain instead of the surface domain. Define a new random variable $\eta$ which represents the surface normal imaged by a pixel. Note that the event $\left(N \in C_{o}\right)$ is not equivalent to the event $\left(\eta \in C_{o}\right)$ because of the effects of masking, shadowing and foreshortening.

\subsubsection{Masking Effects}

Surface points can either be visible or occluded but pixels always correspond to visible surface points. This prior knowledge must be accounted for in deriving $p_{\eta}$ the pdf of the random variable $\eta$. Let the event that a surface point is visible be denoted by $X_{v}$, then

$$
p_{\eta}(n) d n=\operatorname{Pr}(\eta=n)=\operatorname{Pr}\left(N=n \mid X_{v}\right) .
$$

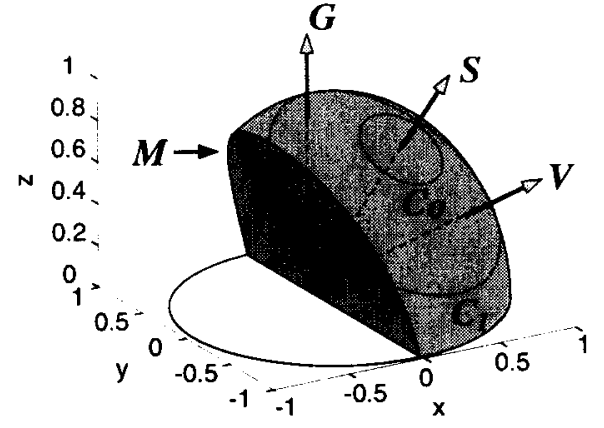

Figure 1. The surface normal set $C_{o}$ is the cone satisfying $\left\{n: n \cdot S=I_{o}\right\}$, where $S$ is the illumination or source direction. Similarly $C_{1}$ is the cone satisfying $\left\{n: n \cdot S=I_{1}\right\}$. The set $M$ of surface normals satisfying $(n \cdot V>0)$ and $(n \cdot G>0)$ is illustrated by the partial sphere. Notice that $C_{o} \cap M$ is the entire cone while $C_{1} \cap M$ is a portion of the cone.

Using Baye's rule,

$$
p_{\eta}(n) d n=\operatorname{Pr}\left(X_{v} \mid N=n\right) \frac{\operatorname{Pr}(N=n)}{\operatorname{Pr}\left(X_{v}\right)}
$$

From simple geometric considerations, it is apparent that a point with surface normal $n$ such that $(n \cdot V<0)$ is not visible. For points with surface normal $n$ such that $(n \cdot V \geq 0)$, a point can be visible or it can be occluded by a neighboring surface point. According to the distant shadower assumption used by $[1],[9],[6]$, the probability that such a point is visible is equal to a constant $q_{v}$. This result is obtained by assuming that the occluder and the occluded surface point lie at a distance larger than the correlation length of the surface; thus each point has equal probability of being occluded regardless of its local surface normal. This assumption has been verified empirically both for one-dimensional surfaces [2] and two-dimensional surfaces [10].

The value of $q_{v}$ depends on surface roughness, illumination direction and viewing direction. Employing this distant shadower assumption, the conditional probability that a surface point is visible is given by

$$
\operatorname{Pr}\left(X_{v} \mid N=n\right)= \begin{cases}q_{v} & n \in M \\ 0 & \text { otherwise }\end{cases}
$$

where $M$ is the set $\{n: n \cdot V>0, n \cdot G>0\}$ illustrated in Figure 1. The probability that a surface point is visible $\operatorname{Pr}\left(X_{v}\right)$ is then given by

$$
\begin{aligned}
\operatorname{Pr}\left(X_{v}\right) & =\int_{n \in M} \operatorname{Pr}\left(X_{v} \mid N=n\right) p_{N}(n) d n \\
& =q_{v} \int_{M} p_{N}(n) d n
\end{aligned}
$$

where the expression $p_{N}(n) d n$ has been used instead of $p_{N}(\theta, \phi) \sin \theta d \theta d \phi$ for notational simplicity. Plugging 
this expression into Equation 5 gives

$$
p_{\eta}(n)= \begin{cases}\frac{p_{N}(n)}{\int_{M} p_{N}(n) d n} & n \in M \\ 0 & \text { otherwise. }\end{cases}
$$

Defining $p_{I}$ as the pdf of the random variable $I$, the probability of intensity $I_{o}$ can now be written as

$$
\begin{aligned}
p_{I}\left(I_{o}\right) d I_{o} & =\operatorname{Pr}\left(I_{o}\right)=\operatorname{Pr}\left(\eta \in C_{o} \cap M\right) \\
& =\iint_{C_{o} \cap M} p_{\eta}(\theta, \phi) \sin \theta d \theta d \phi .
\end{aligned}
$$

This equation accounts for masking, but neglects shadowing and foreshortening.

\subsubsection{Shadowing Effects}

Pixels image either illuminated or shadowed surface points. Define $X_{i}$ as the event that a surface point is illuminated. Consider the probability of some pixel intensity $I_{o}$ which is brighter than the intensity of a shadow,

$$
\operatorname{Pr}\left(I_{o}\right)=\operatorname{Pr}\left(\begin{array}{c}
(\text { pixel is illuminated }) \\
\operatorname{AND}\left(\eta \in C_{o} \cap M\right)
\end{array}\right) .
$$

But the event that a pixel is illuminated is the same as the event that a surface point is illuminated given it is visible. Similarly, the event that a pixel images a surface normal in the set $\dot{C}_{o} \cap M$ is the same as the event that a surface point has surface normal in this set given it is visible. So, $\operatorname{Pr}\left(I_{o}\right)$ is given by

$$
\begin{aligned}
& \operatorname{Pr}\left(I_{o}\right)=\operatorname{Pr}\left(X_{i}, N \in C_{o} \cap M \mid X_{v}\right) \\
& \quad=\operatorname{Pr}\left(X_{i} \mid N \in C_{o} \cap M, X_{v}\right) \operatorname{Pr}\left(N \in C_{o} \cap M \mid X_{v}\right) \\
& =\frac{\operatorname{Pr}\left(X_{i}, X_{v} \mid N \in C_{o} \cap M\right)}{\operatorname{Pr}\left(X_{v} \mid N \in C_{o} \cap M\right)} \operatorname{Pr}\left(\eta \in C_{o} \cap M\right) .
\end{aligned}
$$

We again use the distant shadower assumption to get the following relation:

$$
\operatorname{Pr}\left(X_{i}, X_{v} \mid N=n\right)=\left\{\begin{array}{ll}
q & \text { if } n \in C \cap M \\
0 & \text { otherwise }
\end{array},\right.
$$

where $C=\{n: n \cdot S>0\}$ and $q$ is a constant which depends on illumination, viewing direction and surface roughness. The derivation of $q$ as given in [9] considers only the plane of incidence; so the histogram model is also restricted to the plane of incidence ${ }^{1}$. Equation 11 becomes

$$
\begin{aligned}
\operatorname{Pr}\left(I_{o}\right) & =\frac{q}{q_{v}} \operatorname{Pr}\left(\eta \in C_{o} \cap M\right) \\
& =\frac{q}{q_{v}} \iint_{C_{o} \cap M} p_{\eta}(\theta, \phi) \sin (\theta) d \theta d \phi .
\end{aligned}
$$

The probability of zero intensity, assumed to be the shadow intensity, is simply

$$
\operatorname{Pr}(I=0)=1-\int_{0}^{1} p_{I}\left(I_{o}\right) d I_{o} .
$$

\footnotetext{
${ }^{1}$ However, this is the only aspect of the model that uses the plane of incidence assumption. So extension of the validity of $q$ to outside the plane of incidence would remove the restriction.
}

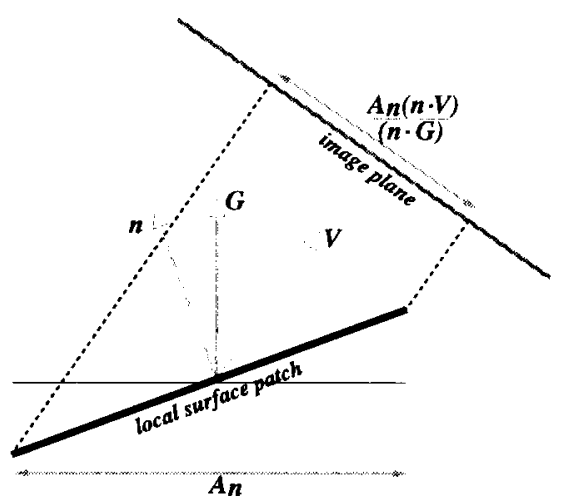

Figure 2. Foreshortening geometry, A section of the surface with normal $n$ and area $A_{n}$ is projected to an image region with area $\frac{A_{n}(n \cdot V)}{(n \cdot G)}$. This geometry is used to derive the foreshortening factor.

\subsubsection{Foreshortening Effects}

Consider a unit area $A_{n}$ with surface normal represented by the vector $n$ or the angles $(\theta, \phi)$. as shown in Figure 2 . This region gets projected to an image region with area $A_{n}(n \cdot V) /(n \cdot G)$, where $V=\left[v_{x}, v_{y}, v_{z}\right]$ is the viewing direction. Therefore, the foreshortening factor is

$$
\frac{(n \cdot V)}{(n \cdot G)}=\tan \theta \cos \phi v_{x}+\tan \theta \sin \phi v_{y}+v_{z} .
$$

After normalization, the pdf $p_{\eta}(\theta . \phi)$ is given by

$$
p_{\eta}(\theta, \phi)=\frac{p_{N}(\theta)}{F}\left(\tan \theta \cos \phi v_{x}+\tan \theta \sin \phi v_{y}+v_{z}\right)
$$

where

$$
F=\iint_{M} p_{N}(\theta)(n \cdot V) /(n \cdot G) \sin \theta d \theta d \phi .
$$

The probability of intensity $I_{o}$ is the probability that the surface normal imaged by a pixel is in the set $C_{o}$, i.e.

$p_{I}\left(I_{o}\right) d I_{o}=\frac{q}{q_{v} F} \iint_{C_{o} \cap M} \frac{p_{N}(\theta \cdot \phi)}{\cos \theta}(n \cdot V) \sin \theta d \phi d \theta$.

This equation takes into account foreshortening, masking and shadowing.

\subsection{Final Form of Histogram Model}

The integral of Equation 17 is quite complicated and does not lend itself to an analytical solution. In order to evaluate the integral we first change the coordinate frame so that the $z$ axis is aligned with the illumination direction $S$ rather than $G$. After this coordinate frame transformation, the polar angle becomes constant and 
the integration is over only one variable. The details of the transformation are given in Appendix A.1. We then project the new integrand onto a set of basis polynomials that are conducive to integration as is detailed in Appendix A.2. As a result of the coordinate transformation and basis decomposition, Equation 17 can be expressed as

$$
{ }_{P I}\left(I_{o}\right) d I_{o}=\frac{q}{q_{v} F} \sum_{j=1}^{L} \alpha_{j} \sum_{i} \kappa_{j}^{i} \mu^{i}
$$

where $L$ is the number of basis polynomials, $\alpha_{j}$ are the coefficients obtained when projecting the integrand onto the basis polynomials, and $\kappa_{j}^{2} \mu^{2}$ are the functions resulting from the integration of the basis polynomials. The details are described in Appendix A.2.

In matrix notation this becomes

$$
{ }_{\text {PI }}\left(I_{o}\right) d I_{o}=\frac{q}{q_{v} F}(\kappa \mu)^{T} \alpha .
$$

where $\kappa$ is an $L x(2 \mathrm{~L}-1)$ matrix which depends on $\mathrm{S}$ (source direction); $\mu$ is a $(2 \mathrm{~L}-1) \times 1$ vector which depends on $V$ (viewing direction) and $\mathrm{S} ; \boldsymbol{\alpha}$ is an $L x$ 1 vector which depends on $\sigma$ (surface roughness); and $\left(\frac{q}{q_{v} F}\right)$ is a constant which depends on $V, S$ and $\sigma$.

Note that $p_{I}\left(I_{0}\right) d I_{0}$ is the value of the histogram for a single intensity bin $I_{o}$. To obtain an expression for the entire histogram, we construct the matrix $\Omega$ so that the rows are the individual $\kappa \mu$ vectors for each intensity $I, \epsilon$ $[0,1]$. For example! if there are 256 discrete intensity values $\Omega$ is a $256 \times L$ matrix. Define $\tilde{h}$ as the predicted histogram vector. Then,

$$
\tilde{h}=\frac{q}{q_{v} F} \Omega \alpha .
$$

This equation gives a simple matrix formula for the complete bidirectional histogram $\tilde{h}$ of a Lambertian, isotropic, randomly rough surface.

\section{Histogram Model Fits}

The measured histograms were obtained from the Columbia-Utrecht texture database for the following samples: Sample 11 (plaster), Sample 10 (plaster), Sample 49 (concrete), Sample 50 (concrete) and Sample 8 (pebbles). For each sample, 19 histograms from images obtained with different viewing and illumination directions were used to represent the measured bidirectional histogram. These histograms correspond to the planeof-incidence measurements from the database. Let $h_{j}(i)$ denote the ith element of the jth measured histogram where $i=\mathbf{0}, 1, . .255$ and $j=\mathbf{0}, 1, \ldots 18$. Similarly let $\tilde{h}_{j}(i)$ denote the ith element of the $j$ th estimated histogram as given by Equation 20. Since our histogram model is appropriate for constant-albedo samples, only gray-scale image information was used. In the model, shadows are assumed to be zero intensity; however, in the actual images shadows are usually non-zero. To account for this discrepancy, all image pixels with intensity lower than a manually chosen shadow threshold are counted as zero-intensity shadows.

The camera response for the measurements was approximately linear so each recorded intensity in related to the actual intensity by a gain factor and an offset. Three parameters were estimated for each sample: gain $\zeta$, offset $\chi$, and roughness parameter $\sigma$. The actual albedo of the sample is implicitly included in the estimation of camera gain $\zeta$. The value for $L$ in Equation 18 was chosen as 16 , for a compact yet accurate representation. The Levenberg-Marquardt algorithm (implemented in Matlab) was used to estimate the parameters $\zeta, \chi$ and $\sigma$ which minimize the error $E$, taken over the collection of histograms, where

$$
E=\sum_{j=0}^{18} \sum_{i=0}^{255}\left(h_{j}(i)-\tilde{h_{j}}(\zeta i+\chi)\right)^{2} .
$$

The fits were improved by applying gaussian blurring to the modeled histogram. The fitting results for five different texture samples are shown in Figure 3. These results indicate a good match between the model and measurements even in the shadow regions that correspond to zero intensity.

\section{Applications}

We now discuss how the model developed in this paper can be used in $3 \mathrm{D}$ texture algorithms for recognition and synthesis.

The model-fitting procedure provides a roughness parameter that can be used for recognition. While all characteristics of the texture are not captured by a single roughness parameter, it does provide a simple concise texture description that is useful for distinguishing a variety of textures. In addition, if roughness and surface slant are known for a portion of the scene, the model can potentially be used to determine the illumination direction. Implementing these applications of recognition and photometric analysis is saved for future work.

This model can also be used in a novel synthesis technique that we have developed for $3 \mathrm{D}$ textures. Assume that the frontal view of the textured surface is given, and the goal is to synthesize an oblique view. Standard texture-mapping addresses this problem by assuming a planar texture. Under this planar assumption, the oblique view is then synthesized by uniformly resampling the frontal view image. For 3D texture, this texture-mapping method results in a rather poor quality synthesis as illustrated in Figure 4 . We introduce a new method of texture-synthesis called texture-morphing which estimates the non-uniform resampling function required to transform a frontal view of 3D texture into an oblique view. This estimation is driven by the goal of matching the histogram of the synthesized texture to the histogram of the oblique view texture. Since the bidirectional histogram model provided in this paper provides 
the target oblique view histogram. only the frontal image and the roughness parameter are required to obtain the oblique view image. At each iteration of the estimation, a comparison is made between the current histogram and the desired histogram; regions where pixels are overrepresented are sampled more sparsely and regions where pixels are underrepresented are sampled more densely. The results of texture-morphing and a comparison to texture-mapping is shown in Figure 4. The synthesis via texture-morphing provides a superior match to the actual oblique view of the sample. suggesting that $3 \mathrm{D}$ texture-rendering can be a promising application of the bidirectional histogram model.

\section{A APPENDIX}

\section{A.1 Coordinate Frame Transformation}

The best coordinate frame to do the integration of Equation 17 is one with the $z$-axis aligned with the illumination direction $\mathrm{S}$. Let $n_{c}$ denote the local surface normal in this coordinate frame with azimuthal angle $\phi_{c}$ and polar angle $\theta_{c}$. Then the integral over the cone is simply the integral over the azimuthal angle $\phi_{c}$. Let $R_{c}$ be the rotation matrix from the coordinate frame aligned with $S$ to the coordinate frame aligned with $G$ so that the conversion of a surface normal $n_{c}$ to $\mathrm{n}$ is given by

$\left.n=R_{c}^{-1} n_{c}=\left[\begin{array}{ccc}\cos \theta_{S} & 0 & \sin \theta_{S} \\ 0 & 1 & 0 \\ -\sin \theta_{S} & 0 & \cos \theta_{S}\end{array}\right] \begin{array}{l}\sin \theta_{c} \cos \phi_{c} \\ \sin \theta_{c} \sin \phi_{c} \\ \cos \theta_{c}\end{array}\right]$

We use the notation

$$
n \cdot V=c \cos \phi_{c}+d \sin \phi_{c}+e,
$$

where $V=\left[v_{x}, v_{y}, v_{z}\right]$ and

$$
\begin{aligned}
\mathrm{C} & =v_{x} \cos \theta_{S} \sin \theta_{c}-v_{z} \sin \theta_{S} \text { sine, } \\
d & =v_{y} \sin \mathrm{e}, \\
\mathrm{e} & =v_{x} \sin \theta_{S} \cos \theta_{c}+v_{z} \cos \theta_{S} \cos \theta_{c}
\end{aligned}
$$

The integral in this coordinate frame is written as

$$
\begin{aligned}
& p_{I}\left(I_{o}\right) d I_{o}= \\
& \frac{q \sin \left(\theta_{c o}\right)}{q_{v} F} \int p_{N}\left(R_{c}^{-1} n_{c o}\right) \frac{\left(c \cos \phi_{c}+d \sin \phi_{c}+e\right)}{\cos \theta\left(\phi_{c}\right)} d \phi_{c} d \theta_{c o} .
\end{aligned}
$$

where $\theta_{c o}$ is the polar angle of the cone corresponding to $I$, in the coordinate frame aligned with $\mathrm{S}, n_{c o}$ is the surface normal defined by $\left(\theta_{c o}, \phi_{c}\right)$ and $\theta\left(\phi_{c}\right)$ is the polar angle of $n_{c o}$ relative to $\mathrm{G}$ as illustrated in Figure 5 .

Because of masking, the integration is generally over only a portion of the cone about $\mathrm{S}$, as illustrated in Figure 1. To compute the limits note that integration is over $M_{v} \cap M_{h}$ where $M_{v}$ is the set $\left\{\phi_{c}: n \cdot V>0\right\}$ and $M_{h}$ is the set $\left\{\phi_{c}: n \mathrm{G}>0\right\}$.

To determine the set $M_{v}$, consider the intersection points where $\mathrm{c} \cos \phi+d \sin \phi+\mathrm{e}=0$, which are

$$
\phi_{\bar{\tau}} \quad 2 \arctan \frac{-2 d+2 \sqrt{\left(d^{2}+c^{2}-e^{2}\right)}}{2(-c+e)},
$$

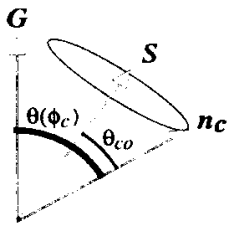

Figure 5. The cone illustrated corresponds to the surface normals for intensity $I_{o}$ according to the Lambertian assumption. In the coordinate frame aligned with the illumination direction $S$, these surface normal have the same polar angle $\theta_{c o}$ but different azimuth angles $\phi_{c}$. A given surface normal $n_{c}$ makes and angle of $\theta\left(\phi_{c}\right)$ with the global surface normal $G$.

$$
\phi_{j}=2 \quad \arctan \frac{-2 d-2 \sqrt{\left(d^{2}+c^{2}-e^{2}\right)}}{2(-c+e)} .
$$

Given these intersections, There are two possibilities for the $M_{v}$. Either

$$
M_{v}=\left\{\phi_{c}: \phi_{i}<\phi_{c}<\phi_{j}\right\}
$$

or

$$
M_{v}=\left\{\phi_{c}: \phi_{j}<\phi_{c}<2 \pi+\phi_{i}\right\}
$$

In order to find the correct choice, the sign of the derivative of $n V$, must be checked at $\phi_{i}$. Specifically, If $\left.(-c \sin \phi+d \cos \phi)\right|_{\phi=\phi_{i}} \geq 0$ then Equation 30 holds; otherwise Equation 31 is the correct choice.

To determine the set $M_{h}$, consider the values of $\phi$ which satisfy

$$
n \cdot \mathrm{G}=\left(-\sin \theta_{S} \sin \theta_{c} \cos \phi+\cos \theta_{S} \cos \mathrm{e},\right)=0
$$

and denote the two solutions as $\phi_{k}$ and $\phi_{l}$, where

$$
\begin{aligned}
& \left.\phi_{k}=\arccos \left(\cos \theta_{S}\right) \frac{\cos \theta_{c}}{\sin \theta_{S} \sin \theta_{c}}\right) \cdot \text { and } \\
& \phi_{l}=-\quad \arccos \left(\left(\cos \theta_{S}\right) \frac{\cos \theta_{c}}{\sin \theta_{S} \sin \theta_{c}}\right) .
\end{aligned}
$$

Checking the derivative of $n . \mathrm{G}$, we find that $M_{h}=$ $\left\{\phi_{c}: \phi_{k}<\phi_{c}<\phi_{l}\right\}$ if $\left.\left(\sin \theta_{S} \sin \theta_{c} \sin \phi\right)\right|_{\phi=o_{k}} \geq 0$; otherwise $M_{h}=\left\{\phi: \phi_{l}<\phi<2 \pi+\phi_{k}\right\}$.

From the geometry of the problem, we know that the sets $M_{h}$ and $M_{v}$ will be continuous. Their intersection however may lead to two disjoint sets $\left\{\phi_{1} \leq 0 \leq \phi_{2}\right\}$ and $\left\{\phi_{3} \leq \phi \leq \phi_{4}\right\}$ as illustrated in Figure 6 . The final general form of the integral is

$$
\begin{aligned}
& p_{I}\left(I_{o}\right)=\frac{q \sin \left(\theta_{c o}\right) d \theta_{c o}}{q_{v} F} \times \\
& \left(\begin{array}{cc}
\int_{\phi_{1}}^{\phi_{2}} p_{N}\left(R_{c}^{-1} n_{c}\right) & \frac{\left(c \cos \phi_{c}+d \sin \phi_{c}+e\right)}{\cos \theta\left(\phi_{c}\right)} d \phi_{c}+ \\
\int_{\phi_{3}}^{\phi 4} p_{N}\left(R_{c}^{-1} n_{c}\right) & \frac{\left(c \cos \phi_{c}+d \sin \phi_{c}+e\right)}{\cos \theta\left(\phi_{c}\right)} d \phi_{c}
\end{array}\right) .
\end{aligned}
$$




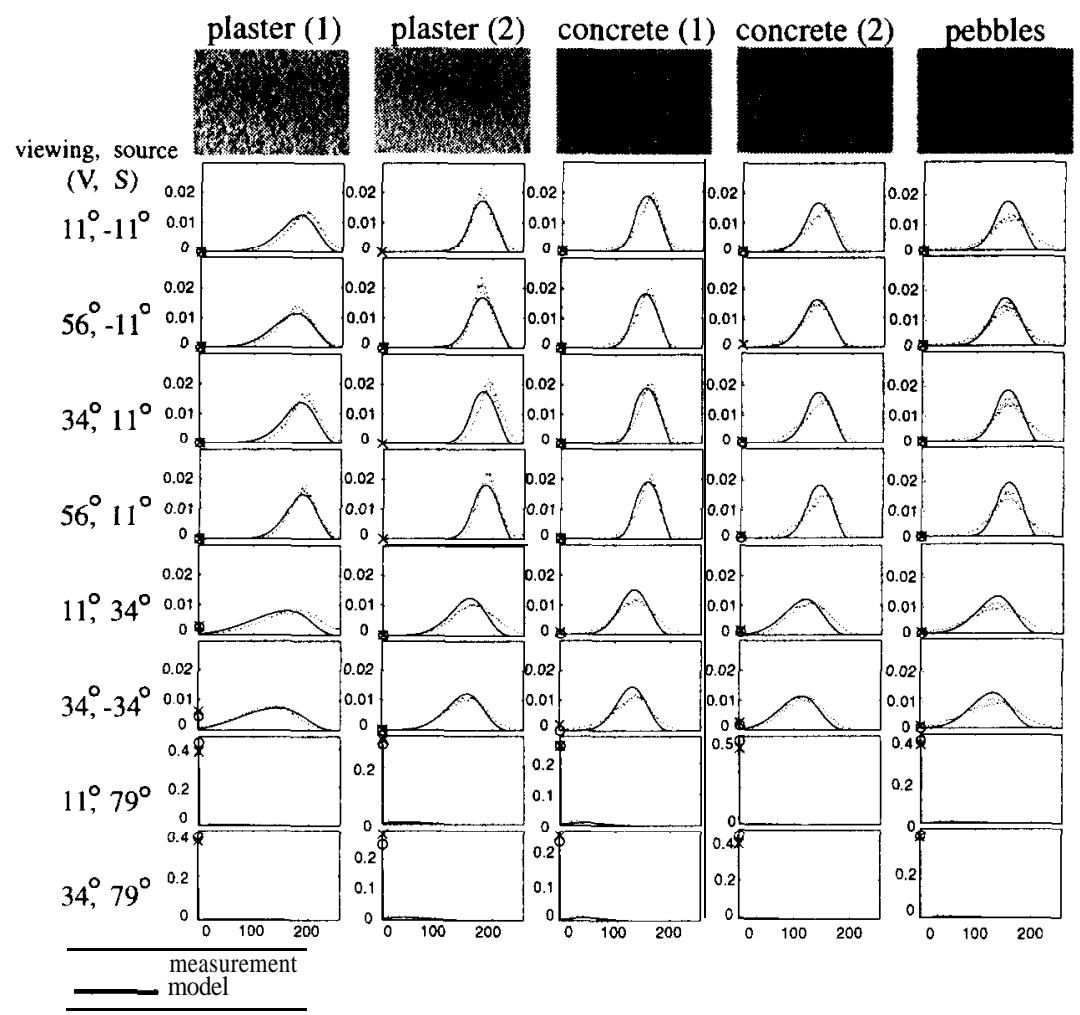

Figure 3. Each column corresponds to the histogram model fit for a sample from the Columbia-Utrecht texture database [3]. From left to right the samples are Sample 11 (plaster), Sample 10 (plaster), Sample 49 (concrete), Sample 50 (concrete) and Sample 8 (pebbles). In each panel the model fit is shown by the solid line while the measured histogram is shown by the dotted line. The zero intensity bin is shown with an ' $x$ ' for the measured histogram and an 'o' for the modeled histogram. The estimated roughness parameter for each sample, from left to right, is 0.41 . 0.23 , $0.24,0.31,0.36$. respectively. For each row, the polar angle of the viewing direction $\mathrm{V}$ and the illumination direction $S$ are given on the left in degrees (negative polar angles correspond to a 180" azimuth). The model was fit using 19 histograms per sample. but for conciseness, 8 histograms per sample are shown in this figure.

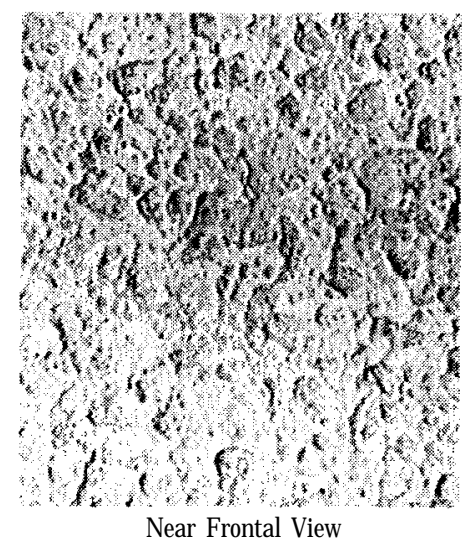

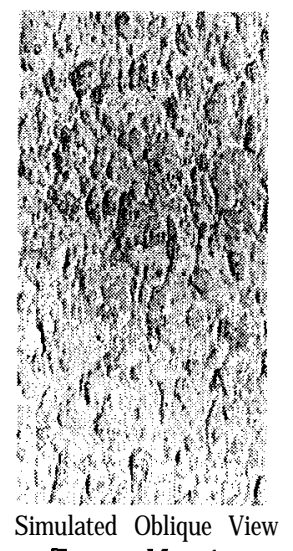

Texture-Mapping

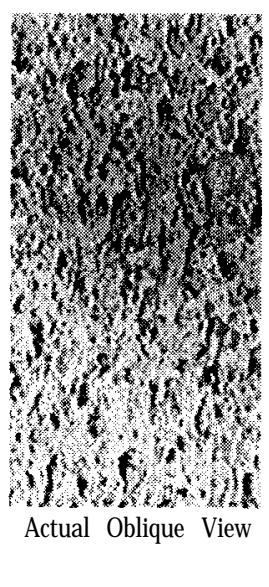

Figure 4. Illustration of $3 \mathrm{D}$ texture synthesis using texture-morphing and the histogram model. From left to right the images are 1) plaster from a near frontal view, 2) a simulated oblique view obtained by applying standard texturemapping to the frontal view image, 3) the actual oblique view of the plaster sample, 4) a simulated oblique view by applying texture-morphing to the frontal view image. The simulated view obtained by texture-morphing is significantly better than texture-mapping. 


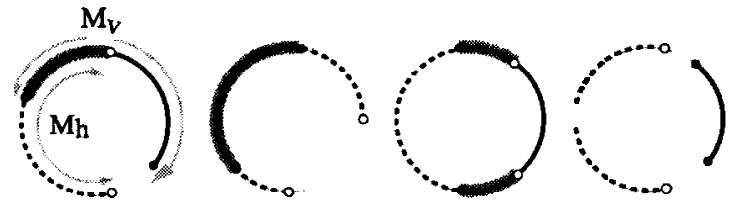

Figure 6. From left to right. the four main intersection outcomes $M_{v} \cap M_{h}$ are illustrated: (1) the sets partially overlap, (2) one set is a subset of the other, (3) the intersection is two disjoint sets. and (4) the intersection is the empty set. In each illustration, the solid line terminated by solid circles corresponds to $M_{v}$, the dashed line terminated by open circles corresponds to $M_{h}$ and the wide gray line corresponds to the intersection.

\section{A.2 Approximation of Integrand}

The integral for $p_{I}$ is very complicated and an analytical solution is not readily attainable. Instead we seek an approximation of the integral. By considering the rotation defined by matrix $R$, we see that

$$
\cos \theta=a \cos \phi_{c}+b
$$

where $\mathrm{a}=-\sin \theta_{S} \sin \theta_{c o}$ and $\boldsymbol{b}=\cos \theta_{S} \cos \theta_{c o}$. An important observation can be made from this relation. Approximations of the integrand in terms of a polynomial in $\cos \theta$ will lead to an approximation that is a polynomial in $\cos \phi_{c}$. Such a polynomial is easily integrated analytically. In fact, it is only necessary to approximate $\left(p_{N}\left(R_{c}^{-1} n_{c}\right) / \cos \theta\right)$ ince the product of a polynomial in $\cos \phi_{c}$ and $\left(\mathrm{c} \cos \phi_{c}+d \sin \phi_{c}+\right.$ e) is also readily integrated analytically. The discrete cosine transform provides us with the means to make this approximation. Defining $\boldsymbol{L}$ as the truncation point of the summation, leads to the following approximation

$$
\begin{aligned}
\frac{p_{N}\left(R_{c}^{-1} n_{c}\right)}{\cos \theta\left(\phi_{c}\right)} & \approx \sum_{j=1}^{L} \alpha_{j} g_{2(j-1)}\left(\cos \theta\left(\phi_{c}\right)\right) \\
& =\sum_{j=1}^{L} \alpha_{j} g_{2(j-1)}\left(a \cos \phi_{c}+b\right),
\end{aligned}
$$

where

$$
g_{j}(\cos \theta)=\cos (j \theta) .
$$

Define $\kappa_{j}^{2}$ to be the coefficient of the term $\cos ^{2} \phi_{c}$ in the polynomial $g_{j}\left(\mathrm{a} \cos \phi_{c}+b\right)$ so that

$$
\frac{p_{N}\left(R_{c}^{-1} n_{c}\right)}{\cos \theta\left(\phi_{c}\right)} \approx \sum_{j} \alpha_{j} \sum_{i} \kappa_{j}^{i} \cos ^{i} \phi_{c} .
$$

Substitute the approximation back into the expression for $p_{I}\left(I_{o}\right)$ in Equation 27, and rearrange to get

$$
\begin{aligned}
& p_{I}\left(I_{o}\right) d I_{o}=\frac{q \sin \left(\theta_{c o}\right)}{q_{v} F} \sum_{j=1}^{L} \alpha_{j} \sum_{i} \kappa_{j}^{i} \times \\
& \int c \cos ^{i+1} \phi_{c}+d \cos ^{\prime \prime} \phi_{c} \sin \phi_{c}+\mathrm{e} \cos ^{2} \phi_{c} d \phi_{c} d \theta_{c} .
\end{aligned}
$$

Then, Equation 35 can expressed as

$$
p_{I}\left(I_{o}\right) d I_{o}=\frac{q}{q_{v} F} \sum_{j=1}^{L} \alpha_{j} \sum_{i} \kappa_{j}^{i} \mu^{i}
$$

where

$$
\begin{aligned}
& \mu^{2}=\int_{\phi_{1}}^{\phi_{2}} \cos ^{2} \phi_{c}\left(c \cos \phi_{c}+d \sin \phi_{c}+\mathrm{e}\right) d \phi_{c} \sin \theta_{c} d \theta_{c} \\
& +\int_{\phi_{3}}^{\phi_{4}} \cos ^{2} \phi_{c}\left(c \cos \phi_{c}+d \sin \phi_{c}+\mathrm{e}\right) d \phi_{c} \sin \theta_{c} d \theta_{c} .
\end{aligned}
$$

In matrix notation this becomes

$$
p_{I}\left(I_{o}\right) d I_{o}=\frac{q}{q_{v} F}(\kappa \mu)^{T} \alpha .
$$

where $\kappa$ is an $L \times(2 L-1)$ matrix which depends on $\mathrm{S}$ (source direction); $\mu$ is a $(2 \mathrm{~L}-1) \times 1$ vector which depends on $V$ (viewing direction) and $\mathrm{S}$ : a is an $L x$ 1 vector which depends on $\sigma$ (surface roughness); and $\left(\frac{q}{q_{v} F}\right)$ is a constant which depends on $V . \mathrm{S}$ and cr.

Construct the matrix $\Omega$ so that the rows are the individual $\kappa \mu$ vectors for each intensity $I_{o} \in[0.1]$. For example, if there are 256 discrete intensity values $\Omega$ is a $256 \times L$ matrix. Define $h$ as the predicted histogram vector. Equation 43 gave the expression. for a single histogram bin, or one element of the vector $\bar{h}$. The entire histogram can be expressed by the following matrix equation,

$$
\tilde{h}=\frac{q}{q_{v} F} \Omega \alpha .
$$

This expression is the final form of the bidirectional histogram model.

\section{References}

[1] P. Beckman, "Shadowing of Random Rough Surfaces." IEEE Trans. Antennas Propag., p. 384, vol. 13. 1965.

[2] R. Brockelman and T. Hagfors, "Note on the effect of Shadowing on the Backscattering of Waves from a random rough surfaces," IEEE Trans. Antennas Propag. vol. 14, p. 621, 1966.

[3] K. Dana, B. van Ginneken, S. N ayar, and J. Koenderink, "Reflectance and Texture of Real-World Surfaces," IEEE Conj. on CVPR, p. 151, 1997.

[4] J.J. Koenderink and A.J. van Doorn, "Illuminance texture due to surface mesostructure," J. Optical Soc. Am. $A$, vol. 13 p. 452, 1996.

[5] T. Leung and J. Malik, "On perpendicular texture or: Why do we see more flowers in the distance," IEEE Conference on CVPR, p. 807, 1997.

[6] B. Smith, "Geometrical Shadowing of Randomly Rough Surfaces," IEEE Trans. Antennas Propag.. p. 668, vol. $15,1967$.

[7] M. Stavridi and J.J. Koenderink, "Surface bidirectional reflection distribution function and the texture of bricks and tiles," Applied Optics, vol. 36, no. 16. p. 3717, 1997.

[8] B. van Ginneken, J.J. Koenderink, and K. Dana, "Texture histograms as a function of irradiaion and viewing direction," IJCV-submitted.

[9] R. Wagner, "Shadowing of Randomly Rough Surfaces," J. Acoust. Soc. Am., p. 138, vol. 41, 1967.

[10]P. Welton, K. Hawker, and H. Frey, "Experimental shadowing measurements on randomly rough surfaces," Journal of the Acoustical Society of America, vol. 54, no. 2, p. 446, 1973. 